\title{
Sensitization of hepatocellular carcinoma cells to irradiation by miR-34a through targeting lactate dehydrogenase-A
}

\author{
XIAOGANG LI ${ }^{1 *}$, PING LU ${ }^{2 *}$, BO LI ${ }^{1}$, RONG YANG ${ }^{1}$, YAN CHU $^{1}$, ZHIPING ZHANG ${ }^{1}$, \\ HONGWEI WAN $^{1}, \mathrm{CHAO}^{2}{ }^{1}$, CHUNXIAO WANG ${ }^{1}$ and KAIYUAN LUO ${ }^{1}$ \\ Departments of ${ }^{1}$ General Surgery and ${ }^{2}$ Vascular Surgery, \\ The Second People's Hospital of Yunnan Province, Fourth Affiliated Hospital of Kunming Medical University, \\ Kunming, Yunnan 650021, P.R. China
}

Received January 7, 2015; Accepted October 14, 2015

DOI: $10.3892 / \mathrm{mmr} .2016 .4974$

\begin{abstract}
Radiation is a therapeutic strategy for the treatment of cancer, and is also used for the treatment of hepatocellular carcinoma. MicroRNAs (miRs) are endogenous, non-coding single-stranded RNA molecules, which regulate gene expression at the post-transcriptional level. In the present study, the roles of miR-34a-mediated glycolysis in radiation sensitivity were investigated. By establishing a radioresistant liver cancer cell line, the present study compared the expression level of miR-34a from radiosensitive and radioresistant cells using the reverse transcription-quantitative polymerase chain reaction. The glucose uptake and lactate production were also compared between the two types of cells. The results demonstrated that miR-34a acted as a tumor suppressor in human hepatocellular cancer cells. Following comparison of radiosensitive and radioresistant cancer cells, the results of the present study demonstrated that miR-34a was negatively correlated with radiation resistance; and levels of miR-34a were significantly downregulated in the HepG2 radioresistant cells. Furthermore, the rate of glycolysis in the radioresistant cells was elevated, and there was evidence that glucose uptake and lactate production increased. Lactate dehydrogenase A (LDHA), which is a key enzyme in the glycolysis signaling pathway, was found to be a target of miR-34a in hepatocellular cancer cells. Notably, the overexpression of miR-34a re-sensitized HepG2 radioresistant cells to radiation treatment by inhibiting LDHA. The results of the present study revealed a negative correlation between miR-34a and glycolysis, caused
\end{abstract}

Correspondence to: Dr Kaiyuan Luo, Department of General Surgery, The Second People's Hospital of Yunnan Province, Fourth Affiliated Hospital of Kunming Medical University, 176 Qingnian Road, Kunming, Yunnan 650021, P.R. China

E-mail: kaiyuanluokmmu@163.com

${ }^{*}$ Contributed equally

Key words: radiosensitivity, radioresistance, microRNA-34a, lactate dehydrogenase A, hepatocellular carcinoma, glucose metabolism by the targeting of LDHA-34a, providing a novel mechanism for miR-34a-mediated radioresistance.

\section{Introduction}

Hepatocellular carcinoma is one of the most common types of cancer worldwide, and is the third most common cause of cancer-associated mortality (1). Patients with hepatocellular carcinoma have a poor prognosis, with a 5-year survival rate of only $3-5 \%$ (2). Radiation has been used in cancer therapy for several decades, and has been integrated into one of the treatments strategies for hepatocellular carcinoma (3). Despite initial promising clinical responses, there is increasing evidence that hepatocellular carcinoma can acquire resistance to radiation $(4,5)$. Although the mechanism underlying radioresistance remains to be fully elucidated, several factors affecting the sensitivity of radioresistant cancer cells have been identified, and investigations are continuing to examine combination treatments using novel strategies, which may allow for decreased dosage and enhanced sensitivity to radiation (5).

MicroRNA (miRNA; miR) is non-coding single-stranded RNA of 22 nucleotides in length (6). Although miRNAs are generally downregulated in cancer, several studies have shown that aberrant miRNA expression is correlated with the development and progression of cancer (7-9). It has been reported that miRNAs affect numerous cancer-associated processes, including proliferation, cell cycle control, apoptosis, differentiation, migration and metabolism (8-10). Therefore, miRNAs may be used as biomarkers for diagnosis, and may be vital targets for cancer molecular therapies.

The miR-34 family consists of three closely associated miRNAs; miR-34a, miR-34b and miR-34c (11). The miR-34 members have been reported to be important downstream effectors of the p53 tumor suppressor (11). In addition, canonical p53 target sites are present in the miR-34 promoters (12). miR-34a has been investigated as a key regulator of tumor suppression (13). The reported miR-34a targets include B cell lymphoma 2, Notch1, cyclin D1, cyclin E2, cyclin-dependent kinase 4, MET and sirtuin 1 (14), suggesting that miR-34a may serve as a cancer therapeutic target.

Lactate dehydrogenase-A (LDHA) is one of the predominant isoforms of $\mathrm{LDH}$, regulating the conversion of pyruvate 
to lactate of the cellular glycolytic process (15). Previous studies have demonstrated that the expression of LDHA in cancer cells is associated with radiotherapy sensitivity (16). In addition, LDHA has been demonstrated to contribute to paclitaxel resistance in breast cancer (17), indicating that LDHA may be a therapeutic target for overcoming drug resistance or radioresistance. In the present study, the roles of LDHA in radiation resistance were investigated in order to identify novel mechanisms underlying miR-34a-mediated radioresistance in human hepatocellular cancer.

\section{Materials and methods}

Cell lines and irradiation treatment. HepG2 and Huh7 human hepatocellular carcinoma cell lines, and normal CL-48 hepatocytes were obtained from American Type Culture Collection (Manassas, VA, USA). The cell cultures were maintained in Dulbecco's modified Eagle's medium, supplemented with $100 \mu \mathrm{M}$ non-essential amino acids, $100 \mu \mathrm{M}$ two-fold vitamin solution (all obtained from Thermo Fisher Scientific, Inc., Waltham, MA, USA), 2 mM L-glutamine (Sigma-Aldrich, St. Louis, MO, USA), $1 \mathrm{mM}$ sodium pyruvate, $10 \%$ fetal bovine serum, $50 \mathrm{U} / \mathrm{ml}$ penicillin and $50 \mu \mathrm{g} / \mathrm{ml}$ streptomycin (Flow Labs, Rockville, MD, USA). The cells were cultured at $37^{\circ} \mathrm{C}$ in a humidified atmosphere containing $5 \% \mathrm{CO}_{2}$ and $95 \%$ air. The cells were irradiated at $0,2,4,8,16$ or $20 \mathrm{~Gy}$ with $6 \mathrm{MV}$ X-rays, generated using a Siemens accelerator (KD-2; Siemens AG, Munich, Germany) at an average dose rate of $200 \mathrm{cGy} / \mathrm{min}$. The cells were trypsinized (Sigma-Aldrich) and re-plated at a density of $1 \times 10^{6}$ cells/well in the cell culture dish in the incubator for $16 \mathrm{~h}$ at $37^{\circ} \mathrm{C}$, followed by the downstream analysis. Cells were counted using a TC20 ${ }^{\mathrm{TM}}$ Automated Cell Counter (Bio-Rad Laboratories, Inc., Hercules, CA, USA) according to the manufacturer's protocols.

Establishment of radioresistant cell lines. A radioresistant hepatocellular carcinoma cell line was generated using HepG2 parental cells, by exposing the cells to repeated irradiation for a duration of 3 months. Cells were counted and passaged for culture overnight. Cells were treated with 10 Gy X-ray generated using a Siemens accelerator (KD-2). Cells were cultured in medium prior to the next passage on day 5 . This was repeated every 2 weeks until radioresistant cell sublines were established. Numerous radioresistant cell clones were developed and pooled for the following experiments.

Reagents and antibodies. The antibodies used in the present study were rabbit anti-human polyclonal LDHA (cat. no. 2012; Cell Signaling Technology, Inc., Danvers, MA, USA) and rabbit anti-human polyclonal $\beta$-actin (cat. no. 4967; Cell Signaling Technology, Inc.). The transfection reagent kit containing Invitrogen Lipofectamine ${ }^{\circledR} 2000$ transfection reagent, and Applied Biosystems miRNA precursor (pre-miRNA-34a) and control miRNAs (control-miRNAs) were purchased from Thermo Fisher Scientific, Inc.

Pre-miRNA or anti-miRNA transfection. The miRNA precursors (pre-miRNAs) and miRNA control (control-miRNA) were transfected into the cells using Lipofectamine ${ }^{\circledR} 2000$. At $48 \mathrm{~h}$ post-transfection, the expression levels of miR-34a were quantified using reverse transcription-quantitative polymerase chain reaction (RT-qPCR), and the protein expression levels of LDHA, a target of miR-34a, were determined using western blotting.

Quantification of the expression levels of $m i R-34 a$. For the analysis of miRNA expression levels, RTq-PCR was performed. RNA was isolated from cultured cells using the RNeasy Mini kit (Qiagen GmbH, Hilden, Germany) with an on-column DNase digestion step, according to the manufacturer's protocols. The quality and quantity of total RNA samples was checked by agarose gel electrophoresis and using the Bioanalyzer RNA 6000 Nano assay (Agilent Technologies GmbH, Waldbronn, Germany). The RNA was polyadenylated by poly(A) polymerase (New England BioLabs, Inc., Ipswich, MA, USA) and reverse transcribed into cDNA with a Promega reverse transcription kit (Promega Corporation, Madison, WI, USA) according to the manufacturer's instructions. qPCR was performed in a $20-\mu 1$ reaction containing $1 \mu \mathrm{l}$ TaqMan ${ }^{\circledR}$ Small RNA Assay (20X; Thermo Fisher Scientific, Inc.), $1 \mu \mathrm{l}$ cDNA, $0.5 \mu \mathrm{l}$ miRNA primers $(20 \mathrm{mmol} / \mathrm{l}$; Invitrogen; Thermo Fisher Scientific, Inc.), $10 \mu 1$ TaqMan ${ }^{\circledR}$ Universal PCR Master Mix II (2X; Thermo Fisher Scientific, Inc.) and $7.5 \mu 1$ nuclease-free water. The reaction mixtures were incubated at $95^{\circ} \mathrm{C}$ for 15 min, followed by 40 cycles at $95^{\circ} \mathrm{C}$ for $10 \mathrm{sec}$, at $60^{\circ} \mathrm{C}$ for $30 \mathrm{sec}$ and at $72^{\circ} \mathrm{C}$ for $30 \mathrm{sec}$, running on a Mx3000 $\mathrm{P}^{\mathrm{TM}}$ thermocycler (Agilent Technologies, Inc., Santa Clara, CA, USA). The miRNA primer sequences were as follows: Sense, 5'-CGC TTCGGCAGCACATATACTA-3' and antisense, 5'-CGCTTC ACGAATTTGCGTGTCA-3' for U6 small nuclear RNA (snRNA); and sense, 5'-TTTAAGCTTATGCGCCCTGCC-3' and antisense, 5'-TTTCTCGAGAGAGCTTCCGAAGTC CTGG-3' for miR-34a. Quantitative miRNA expression level data were analyzed by MxPro software (Agilent Technologies, Inc.) and the normalization was performed with U6 snRNA. The miRNA was quantified using the formula: $\triangle$ CqmiRNA = CqmiRNA - CqU6 (12,17). All reactions were performed in triplicate.

Plasmid DNA and small interfering (si)RNA transfection. Transfection was performed using Lipofectamine ${ }^{\circledR} 2000$ transfection reagent, according to the manufacturer's protocol. Overexpression vectors containing wild-type LDHA (cat. no. RC209378) were purchased from Origene Technologies, Inc. (Rockville, MD, USA). The Invitrogen siLDHA and negative control siRNA were purchased from Thermo Fisher Scientific, Inc. At 48 h post-transfection, the cells were collected and the whole-cell lysates were prepared using the Mammalian Protein Extraction reagent (Pierce Biotechnology, Inc., Rockford, IL, USA) for further analysis.

Glucose uptake and lactate product assays. Glucose levels were determined using a Glucose Assay kit (BioVision, Inc., Milpitas, CA, USA), and glucose consumption was calculated as the difference in glucose concentration between the original media and the media from the cell cultures. Absorbance was measured at $563 \mathrm{~nm}$ using a Spectra Max M5 plate reader (Molecular Devices, LLC, Sunnyvale, CA, USA). Extracellular lactate levels were measured in the cell culture media using a Lactate Assay kit (BioVision, Inc.), according to 
the manufacturer's protocol. The results were normalized to the quantity of total protein in the control cells.

Cell viability assay. Following irradiation treatments, $5 \times 10^{4}$ cells were added to each well of the 96-well plates and incubated for $24 \mathrm{~h}$ at $37^{\circ} \mathrm{C}$. The present study used irradiation treatment doses of $0,2,4,8,16$ or 20 Gys. Sensitivities to these radiation treatments were determined using an MTT assay. The incubation medium was removed, and $50 \mu \mathrm{l}$ MTT solution (1 mg/ml; Sigma-Aldrich) was added to each well prior to incubation for a further $4 \mathrm{~h}$. Following incubation at $37^{\circ} \mathrm{C}, 200 \mu \mathrm{l}$ dimethyl sulfoxide (Sigma-Aldrich) was added to each well. The optical density values were measured at $570 \mathrm{~nm}$ using a ELx808 ${ }^{\mathrm{TM}}$ Absorbance Microplate Reader (BioTek Instruments, Inc., Winooski, VT, USA). All experiments were repeated at least twice.

Western blot analysis. Total protein was extracted using Mammalian Protein Extraction reagent and the protein concentration was measured using the Bradford assay (Bio-Rad Laboratories, Inc.). The protein concentrations were measured by reading the absorbance at a wavelength of $595 \mathrm{~nm}$ on the ELx808 ${ }^{\mathrm{TM}}$ Absorbance Microplate Reader. An equal quantity $(50 \mu \mathrm{g})$ of protein from each cell line was loaded per lane and separated on 10\% SDS-PAGE (Bio-Rad Laboratories, Inc.). The gels were electroblotted onto nitrocellulose membranes (Novex, San Diego, CA, USA) and blocked overnight at room temperature with $1 \mathrm{X}$ Tris-buffered saline with $0.1 \%$ Tween (Bio-Rad Laboratories, Inc.) and $4 \%$ non-fat dry milk. The membranes were then probed with primary antibodies against LDHA or $\beta$-actin at 1:1,000 dilution at $37^{\circ} \mathrm{C}$. The bound antibodies were then detected using goat anti-rabbit polyclonal peroxidase-coupled secondary antibodies (1:3,000; Cell Signaling Technology, Inc.; cat. no. 7074 ) for $1 \mathrm{~h}$ at $37^{\circ} \mathrm{C}$ prior to detection with a Super Signal Enhanced Chemiluminescence kit (Pierce Biotechnology, Inc.).

Prediction of miR-34a targets. The putative targets of miR-34a were predicted from the public miRNA database, TargetScan (www.targetscan.org) by searching the 3'-untranslated region of mRNA, which contains a highly conserved binding site for miR-34a.

Statistical analysis. Statistical evaluation for data analysis was determined using an unpaired Student's t-test. Student's t-test was calculated using GraphPad Prism 5.0 software (GraphPad Software, Inc., La Jolla, CA, USA) to assess for statistical differences. All data are presented as the mean \pm standard error of the mean. $\mathrm{P}<0.05$ was considered to indicate a statistically significant difference.

\section{Results}

miR-34a is downregulated in human hepatocellular carcinoma cells and correlates with radiation sensitivity. As previous studies demonstrated that miR-34a acts as a tumor suppressor in several types of tumor (11-14), the present study examined the expression levels of miR-34a in human hepatocellular carcinoma cells, which were then compared with normal CL-48 hepatocyte cells. As expected, the expression levels of miR-34a were significantly downregulated in the hepatocellular carcinoma cell lines, compared with the normal hepatocyte cell line (Fig. 1A), suggesting miR-34a may act as a tumor suppressor in hepatocellular carcinoma cells. The roles of miR-34a in radiation sensitivity were also investigated. The cells were transfected with pre-miR-34a for $48 \mathrm{~h}$, followed by exposure to radiation treatments between 0 and 6 Gy. Overexpression of miR-34a in the HepG2 and Huh-7 cells (Fig. 1B) significantly decreased cell viability, compared with the control miRNA (Fig. 1C), indicating the potential for miR-34a to be applied clinically to enhance the radiosensitivity of hepatocellular carcinoma.

Radioresistant hepatocellular carcinoma cells exhibit lower expression levels of miR-34a. The correlation between the expression of miR-34a and radioresistance in cancer cells remains to be fully elucidated. To investigate the mechanisms underlying miR-34a-mediated radiation sensitivity, a radioresistant hepatocellular carcinoma cell line was generated using HepG2 parental cells, by exposing the cells to repeated irradiation for a duration of 3 months. Numerous radioresistant cell clones were developed and pooled for the following experiments. To verify radioresistance, the parental cells and radioresistant cells were treated with various doses of radiation(2-20 Gy). Subsequent cell viability assays demonstrated that the radioresistant HepG2 cells tolerated treatment with higher doses of radiation, compared with the parental (sensitive) cells, which exhibited a significant inhibition in viability between 4 and 20 Gy (Fig. 2A). As expected, the expression levels of miR-34a in the HepG2 radioresistant cells were significantly downregulated (Fig. 2B), compared with the parental cells, indicating that miR-34a may serve as a therapeutic agent for selection in the treatment of radioresistant cancer patients.

Glucose metabolism is upregulated in radioresistant HepG2 cells through the induction of the expression of LDHA. It has previously been reported that enhanced aerobic glycolysis causes acquired radioresistance in human tumor cells (18), indicating that dysregulated glucose metabolism may contribute to radioresistance. In the present study, glucose uptake and lactate production were measured, which reflected the activity of glycolysis. As shown in Fig. 3A and B, glucose uptake and lactate production in the HepG2 radioresistant cells were upregulated, compared with the parental cells. LDHA catalyzes the interconversion between pyruvate and L-lactate, which is further secreted from the cells (15). Therefore, the upregulation of LDHA may serve as a marker for increased glycolysis in cancer cells (15). Treatments with radiation at low doses ( 0.1 and $0.5 \mathrm{~Gy})$ induced the expression of LDHA in the HepG2 cells (Fig. 3C). Concordantly, the expression of LDHA was upregulated in the radioresistant HepG2 cells (Fig. 3D), indicating that LDHA was involved in the radioresistance of hepatocellular carcinoma cells. To investigate whether the increase in glycolysis in the radioresistant HepG2 cells occurred via the increased expression levels of LDHA, the expression of LDHA was knocked down by siRNA to monitor the rate of glycolysis. As expected, the levels of glucose uptake and lactate production were lower in the LDHA-knockdown radioresistant HepG2 cells (Fig. 3E and F). 
A

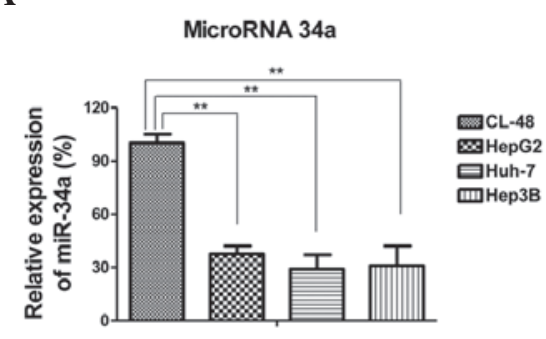

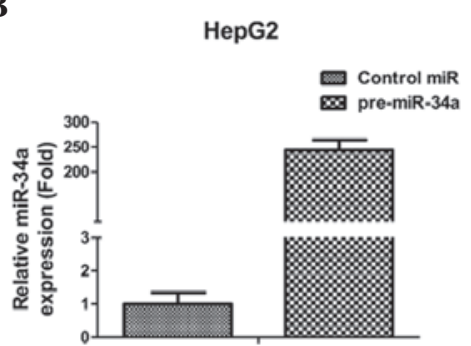

Huh-7

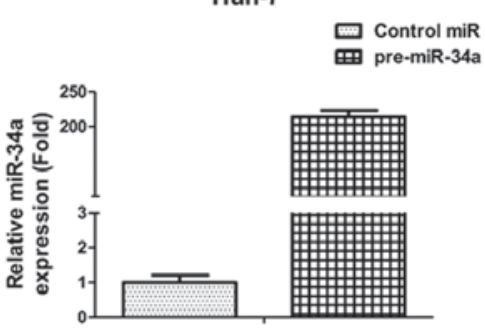

C

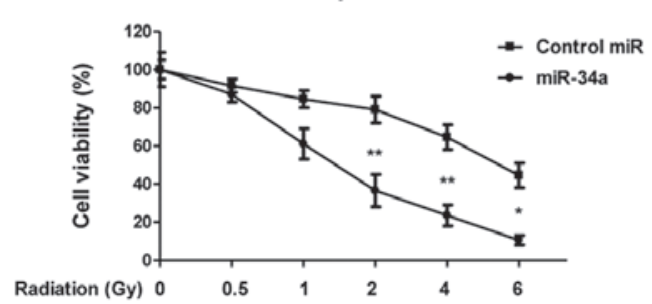

HepG2
Figure 1. miR-34a acts as a tumor suppressor in hepatocellular cancer cells. (A) Expression of miR-34 in HepG2, Huh-7 and Hep3B hepatocellula carcinoma cell lines, compared with normal CL-48 human hepatocyte cell line. (B) Overexpression of miR-34a in Huh-7 (left) and HepG2 (right) cells. (C) Overexpression of miR-34a in Huh-7 (left) and HepG2 (right) cells increased the sensitivity to radiation. Cells were transfected with scramble control microRNA or pre-miR-34a for $48 \mathrm{~h}$, following which the cells were then treated by irradiation at multiple dosages and cell viability analysis was performed. Columns represent the mean of three independent experiments and data are presented as the mean \pm standard error of the mean. ${ }^{*}<0.05$ and ${ }^{* * *} \mathrm{P}<0.01$ vs. the control. miR, microRNA.

LDHA is a target of miR-34a in hepatocellular carcinoma cells. To further examine the potential association between dysregulated expression levels of miR-34a and increased glycolysis in radioresistant hepatocellular carcinoma cells, an miRNA database was accessed to identify potential miR-34a targets that may contribute to radiation resistance. The public miRNA database, TargetScan, predicted that LDHA may be a target of miR-34a, and that the 3'-untranslated region of LDHA contains a highly conserved binding site for miR-34a (Fig. 4A). To determine whether miR-34a targets LDHA in human hepatocellular carcinoma cells, pre-miR-34a was transfected into HepG2 and Huh-7 cells. The overexpression of miR-34a markedly downregulated the protein expression levels of LDHA in the two cell lines (Fig. 4B), indicating that miR-34a-mediated radiosensitivity may act through the inhibition of glycolysis by targeting LDHA.

Overexpression of $\mathrm{miR}-34$ a resensitizes radioresistant $\mathrm{Hep} G 2$ cells to radiation through the inhibition of $L D H A$. The data described above revealed a potential role for miR-34a in radioresistance and the regulation of glycolysis. Therefore, the present study hypothesized that the downregulation of LDHA by the overexpression of miR-34a in hepatocellular carcinoma cells may resensitize radioresistant cells to radiation treatment. To confirm this hypothesis, the HepG2 radioresistant cells were transfected with pre-miR-34a and, after $48 \mathrm{~h}$, the cells were subjected to radiation treatment at various concentrations for $24 \mathrm{~h}$. As shown in Fig. 5A, the overexpression of miR-34a significantly resensitized the resistant cells to radiation treatment. In addition, glucose uptake (Fig. 5B left) and lactate production (Fig. 5B right) were decreased following transfection of the HepG2 radioresistant cells with miR-34a. To further support these results, the expression of LDHA
$\mathbf{A}$

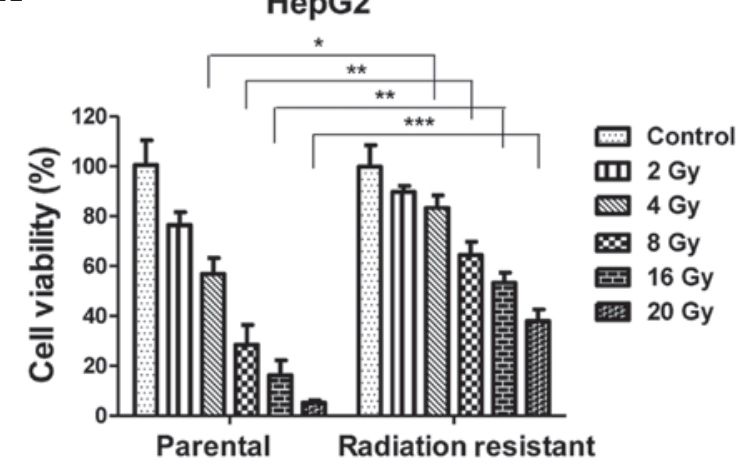

B

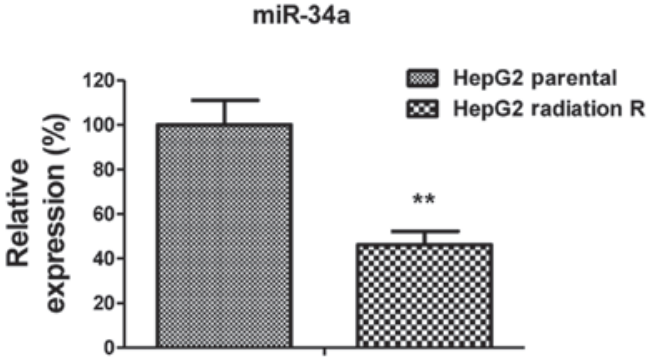

Figure 2. Expression of miR-34a is negatively correlated with radiosensitivity. (A) Generation of radioresistant cells from HepG2 cells. The cells were treated with gradually increasing dosages of radiation for 3 months in order to select for radioresistant cells. Radioresistant clones were pooled and exposed to radiation at 2, 4, 8, 16 and 20 Gys, prior to analysis using a cell viability assay. (B) Expression levels of miR-34a in HepG2 parental cells and radioresistant cells. Columns represent the mean of three independent experiments. Data are presented as the mean \pm standard error of the mean. ${ }^{*} \mathrm{P}<0.05,{ }^{* *} \mathrm{P}<0.01$ and ${ }^{* * *} \mathrm{P}<0.001 . \mathrm{miR}$, microRNA.

was recovered by transfecting the miR-34a pre-transfected radioresistant cells with an LDHA expression vector (Fig. 5C). 


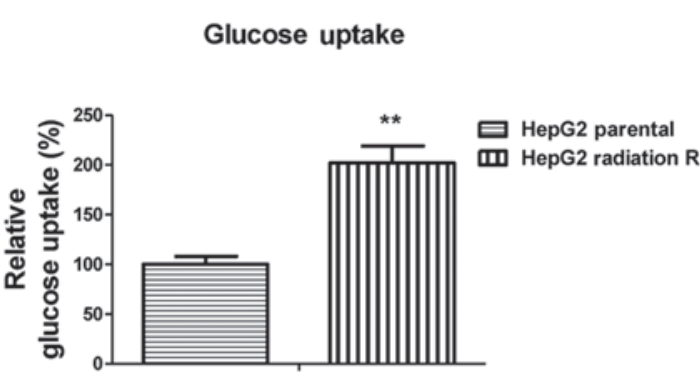

C

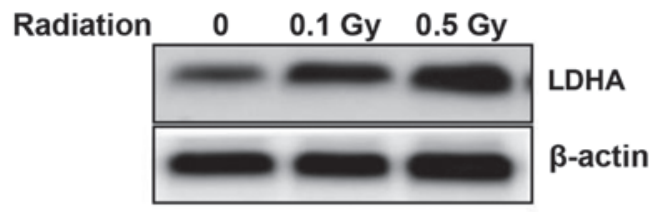

D

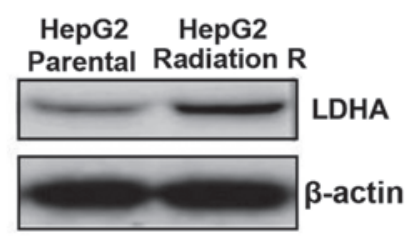

$\mathbf{E}$

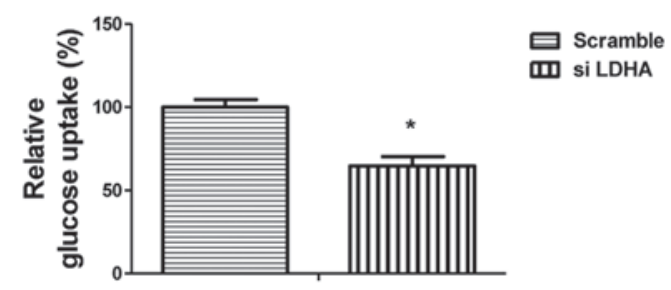

B

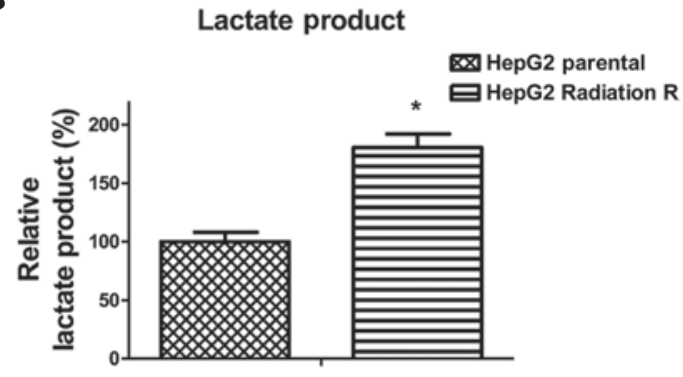

LDHA

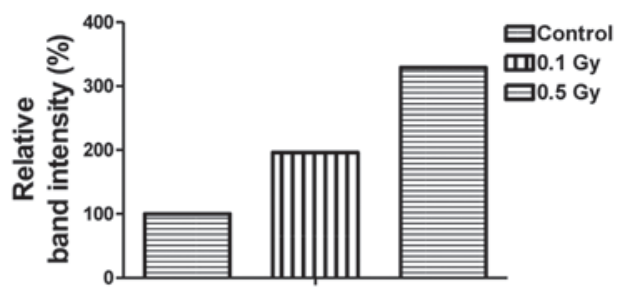

LDHA

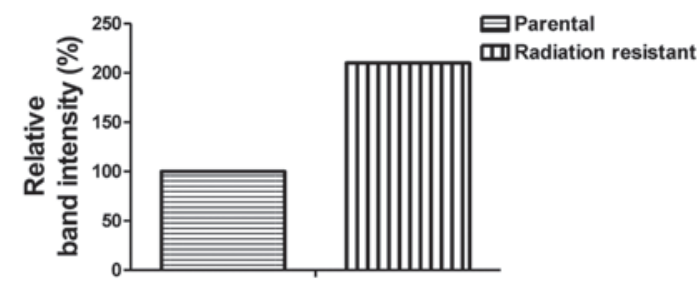

F

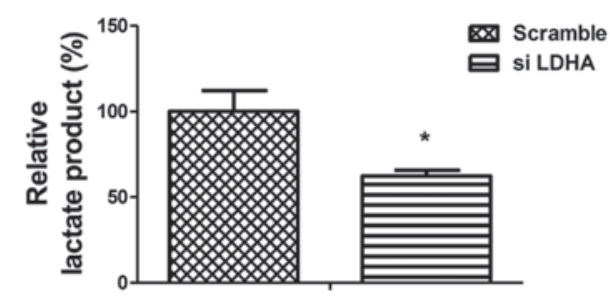

Figure 3. Radioresistant hepatocellular carcinoma cells exhibit elevated glucose metabolism through the upregulation of LDHA. (A) Glucose uptake and (B) lactate production were measured in HepG2 parental cells and radioresistant HepG2 cells. (C) Western blotting revealed that the expression levels of LDHA in the HepG2 cells were induced by radiation at low doses. The relative expression was determined by the band intensity. (D) Western blotting revealed that the expression levels of LDHA in HepG 2 radioresistant cells were higher, compared with those in the parental cells. $\beta$-actin was used as a loading control. The relative expression was determined by the band intensity. HepG2 radioresistant cells were transfected with siLDHA or scramble control for $48 \mathrm{~h}$ prior to the measurement of (E) glucose uptake and (F) lactate production. Columns represent the mean of three independent experiments. Data are presented as the mean \pm standard error of the mean. ${ }^{*} \mathrm{P}<0.05$ and ${ }^{* *} \mathrm{P}<0.01$. LDHA, lactate dehydrogenase A; siLDHA, small interfering LDHA.

The recovery of LDHA increased the resistance of the HepG2 cells to radiation (Fig.5D), indicating that miR-34a-mediated radiosensitivity occurred through the inhibition of LDHA.

\section{Discussion}

miR-34a has been recognized as a key regulator of tumor suppression (11-14). Furthermore, several studies have reported that miR-34a modulates anticancer drug responses in multiple types of cancer. It has been reported that the ectopic overexpression of miR-34a increases sensitivity to doxorubicin (19), 5-fluorouracil (20), Erlotinib (21) and Taxol (22) treatment. In the present study, miR-34a was found to be involved in the resistance of human hepatocyte cancer cells to radiation. The expression of miR-34a was downregulated in human hepatocyte cancer cell lines, compared with normal hepatocytes. In addition, the overexpression of miR-34a contributed to radioresistance, which was concordant with previous reports demonstrating that miR-34a acts as a tumor suppressor and confers chemosensitivity (11-14).

The majority of cancer cells rely on aerobic glycolysis to generate the energy required for cellular processes. By contrast, normal differentiated cells rely primarily on mitochondrial oxidative phosphorylation (23). Therefore, dysregulated anaerobic glycolysis has been associated with drug resistance and radioresistance in cancer (23). It has been reported that LDHA contributes to paclitaxel (17) and trastuzumab (24) resistance. Furthermore, radiation induces aerobic glycolysis through 
hsa-miR-34a

Position 406-413 of LDHA 3' UTR 5' ...CCACCUCUGACGCACCACUGCCA...

B

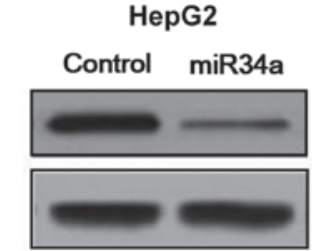

$\beta$-actin

HepG2

LDHA

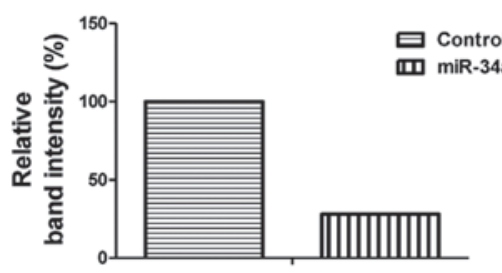

UguUggucgauUCugugacggu

Huh-7

Control miR34a

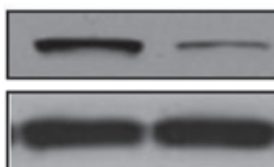

Huh-7

LDHA

seed match

8mer

LDHA

$\beta$-actin

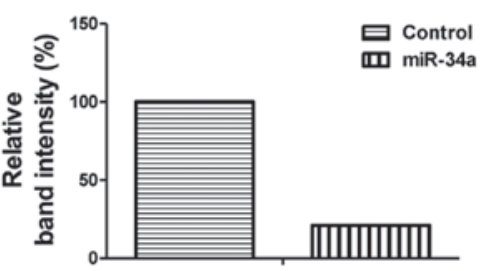

Figure 4. LDHA is a target of miR-34a in hepatocellular carcinoma cells. (A) Target prediction from TargetScan predicted position 406-413 of the LDHA 3'-UTR to contain putative binding sites for miR-34a. (B) HepG2 cells (left) and Huh-7 cells (right) were transfected with $100 \mathrm{nM}$ pre-miR-negative (control) or pre-miR-34a for $48 \mathrm{~h}$. Cell lysates were prepared for western blot analysis and relative expression was determined from the band intensity. $\beta$-actin was used as a loading control. Overexpression of miR-34a in HepG2 and Huh-7 cells inhibited the LDHA protein expression. LDHA, lactate dehydrogenase A; miR, microRNA; UTR, untranslated region.

A

HepG2

radiation resistant

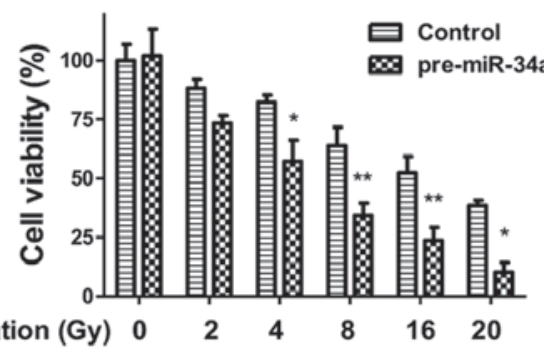

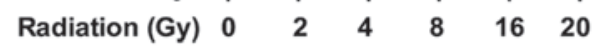

B

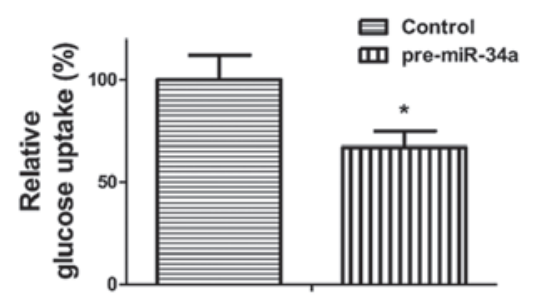

Lactate product

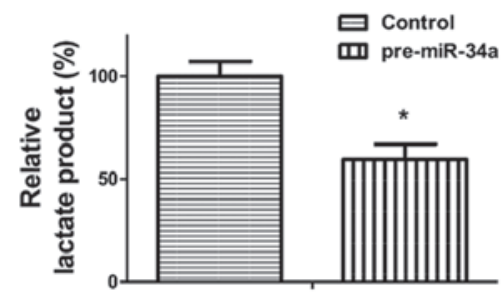

C

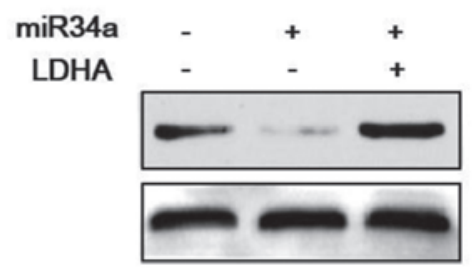

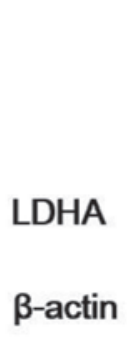

$\mathbf{D}$

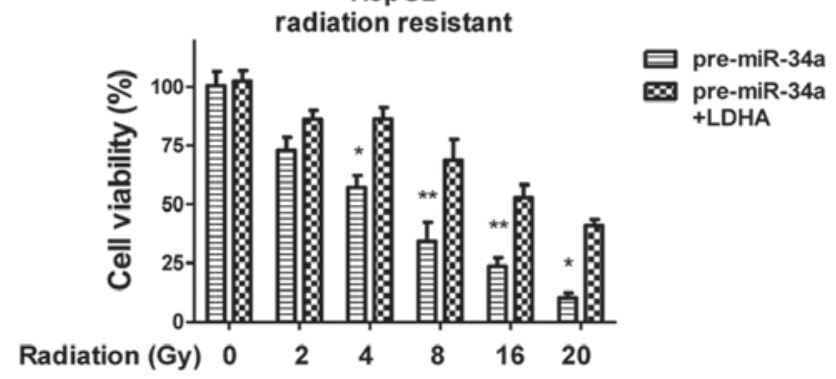

Figure 5. Overexpression of miR-34a in HepG2 radioresistant cells enhances radiosensitivity. (A) HepG2 radioresistant cells were transfected with scramble control siRNA or per-miR-34a for $48 \mathrm{~h}$, and the cells were then exposed to radiation $(0,2,4,8,16$ and 20 Gys) prior to cell viability analysis. (B) Overexpression of miR-34a in the radioresistant HepG2 cells inhibited glucose metabolism. (C) Radioresistant HepG2 cells were transfected with pre-miR-34a or control siRNA for $48 \mathrm{~h}$, following which the miR-34a-overexpressing cells were transfected with a control vector or an overexpression vector containing LDHA for $24 \mathrm{~h}$. The cells were then subjected to western blot analysis. $\beta$-actin was used as a loading control. " $\mathrm{P}<0.05$ and ${ }^{* *} \mathrm{P}<0.01$ vs. the control. (D) Cells were transfected with pre-miR-34a for $48 \mathrm{~h}$, and transfected with a control vector or an overexpression vector containing LDHA for $48 \mathrm{~h}$. Cells were treated with radiation $(0,2,4,8,16$ and 20 Gys), prior to cell viability analysis. " $\mathrm{P}<0.05$ and $^{* *} \mathrm{P}<0.01$ vs. the pre-miR-34a + LDHA of each treatment. Columns represent the mean of three independent experiments. Data are presented as the mean \pm standard error of the mean. siRNA, small interfering RNA; miR, microRNA; LDHA, lactate dehydrogenase A.

reactive oxygen species (25), suggesting that cancer cells with active glycolysis may acquire radioresistance. Currently, as a clinically relevant area in cancer research, the dysregulation of glycolysis and radiosensitivity has not been specifically 
addressed. The results of the present study demonstrated that radioresistant cancer cells exhibit upregulated levels of glycolysis and downregulated expression levels of miR-34a. To the best of our knowledge, the present study is the first to describe a direct association between glycolysis and radioresistance in human liver cancer. A TargetScan database search in the present study identified LDHA as a target of miR-34a, and the results demonstrated that overexpression of miR-34a inhibited glycolysis by targeting LDHA, leading to sensitization of radioresistant cancer cells to radiation.

Radiation therapy generates free radicals, which damage DNA in order to induce cancer cell apoptosis. Generally, ionizing radiation induces reactive oxygen species in targeted cells, leading to genomic instability and lipid peroxidation (26). Of note, excessive lactate produced by cancer cells has been reported to be an antioxidant, which may counteract the reactive oxygen species generated by irradiation (27). Therefore, it is possible that the accumulation of lactate surrounding a tumor may contribute to its radioresistance. The results of the present study demonstrated a significant correlation between the expression of LDHA and radioresistance. The inhibition of LDHA by miR-34a resensitized the radioresistant cells to irradiation, and the restoration of LDHA in the miR-34a-overexpressing cells reversed the resensitization, suggesting that targeting LDHA may be a therapeutic strategy for the treatment of radiation resistance. Future investigation aim to identify potential glycolysis inhibitors, in order to investigate whether the administration of these inhibitors in combination with radiation treatments exhibits synergistic effects on hepatocellular carcinoma cells. In addition, the use of a mouse model offers potential in verifying the phenotypes.

In conclusion, the present study demonstrated a marked correlation between miR-34a-mediated glycolysis and radiation resistance, and provides novel insights into the role of LDHA in anti-radioresistance therapy.

\section{Acknowledgements}

The authors of the present study would like to thank the staff and the faculty members working in the Department of General Surgery and the Department of Vascular Surgery, The Second People's Hospital of Yunnan Province and Fourth Affiliated Hospital of Kunming Medical University (Kunming, China). The present study was supported by funding from the Departments of General Surgery and Vascular Surgery, The Second People's Hospital of Yunnan Province and Fourth Affiliated Hospital of Kunming Medical University.

\section{References}

1. Karaman B, Battal B, Sari S and Verim S: Hepatocellular carcinoma review: Current treatment, and evidence-based medicine. World J Gastroenterol 20: 18059-18060, 2014.

2. Olsen SK, Brown RS and Siegel AB: Hepatocellular carcinoma: Review of current treatment with a focus on targeted molecular therapies. Therap Adv Gastroenterol 3: 55-66, 2010.

3. Hong TS: Radiotherapy for hepatocellular carcinoma with tumor vascular thrombus: Ready for prime time? J Clin Oncol 31: 1619-1620, 2013.

4. Singh S, Singh PP, Roberts LR and Sanchez W: Chemopreventive strategies in hepatocellular carcinoma. Nat Rev Gastroenterol Hepatol 11: 45-54, 2014.
5. Klein J and Dawson LA: Hepatocellular carcinoma radiation therapy: Review of evidence and future opportunities. Int J Radiat Oncol Biol Phys 87: 22-32, 2013.

6. Ha M and Kim VN: Regulation of microRNA biogenesis. Nat Rev Mol Cell Biol 15: 509-524, 2014.

7. Jansson MD and Lund AH: MicroRNA and cancer. Mol Oncol 6: 590-610, 2012.

8. Nelson KM and Weiss GJ: MicroRNAs and cancer: Past, present and potential future. Mol Cancer Ther 7: 3655-3660, 2008.

9. Bouyssou JM, Manier S, Huynh D, Issa S, Roccaro AM and Ghobrial IM: Regulation of microRNAs in cancer metastasis. Biochim Biophys Acta 1845: 255-265, 2014.

10. Dumortier O, Hinault C and Van Obberghen E: MicroRNAs and metabolism crosstalk in energy homeostasis. Cell Metab 18: 312-324, 2013.

11. Misso G, Di Martino MT, De Rosa G, Farooqi AA, Lombardi A, Campani $\mathrm{V}$, Zarone MR, Gullà $\mathrm{A}$, Tagliaferri $\mathrm{P}$, Tassone $\mathrm{P}$ and Caraglia M: Mir-34: A new weapon against cancer? Mol Ther Nucleic Acids 3: e194, 2014.

12. Chang TC, Wentzel EA, Kent OA, Ramachandran K, Mullendore M, Lee KH, Feldmann G, Yamakuchi M, Ferlito M, Lowenstein CJ, et al: Transactivation of miR-34a by p53 broadly influences gene expression and promotes apoptosis. Mol Cell 26: 745-752, 2007.

13. Cole KA, Attiyeh EF, Mosse YP, Laquaglia MJ, Diskin SJ, Brodeur GM and Maris JM: A functional screen identifies miR-34a as a candidate neuroblastoma tumor suppressor gene. Mol Cancer Res 6: 735-742, 2008.

14. Li XJ, Ren ZJ and Tang JH: MicroRNA-34a: A potential therapeutic target in human cancer. Cell Death Dis 5: e1327, 2014.

15. Miao P, Sheng S, Sun X, Liu J and Huang G: Lactate dehydrogenase A in cancer: A promising target for diagnosis and therapy. IUBMB Life 65: 904-910, 2013.

16. Hennessey D, Martin LM, Atzberger A, Lynch TH, Hollywood D and Marignol L: Exposure to hypoxia following irradiation increases radioresistance in prostate cancer cells. Urol Oncol 31: 1106-1116, 2013.

17. Zhou M, Zhao Y, Ding Y, Liu H, Liu Z, Fodstad O, Riker AI, Kamarajugadda S,Lu J, Owen LB, et al: Warburg effect in chemosensitivity: Targeting lactate dehydrogenase-A re-sensitizes taxol-resistant cancer cells to taxol. Mol Cancer 9: 33, 2010.

18. Shimura T, Noma N, Sano Y, Ochiai Y, Oikawa T, Fukumoto M and Kunugita N: AKT-mediated enhanced aerobic glycolysis causes acquired radioresistance by human tumor cells. Radiother Onco 112: 302-307, 2014.

19. Li XJ, Ji MH, Zhong SL, Zha QB, Xu JJ, Zhao JH and Tang JH: MicroRNA-34a modulates chemosensitivity of breast cancer cells to adriamycin by targeting Notch1. Arch Med Res 43: 514-521, 2012.

20. Akao Y, Noguchi S, Iio A, Kojima K, Takagi $\mathrm{T}$ and Naoe T: Dysregulation of microRNA-34a expression causes drug-resistance to 5-FU in human colon cancer DLD-1 cells. Cancer Lett 300: 197-204, 2011.

21. Zhao J, Kelnar K and Bader AG: In-depth analysis shows synergy between erlotinib and miR-34a. PLoS One 9: e89105, 2014.

22. Kojima K, Fujita Y, Nozawa Y, Deguchi T and Ito M: MiR-34a attenuates paclitaxel-resistance of hormone-refractory prostate cancer PC3 cells through direct and indirect mechanisms. Prostate 70: 1501-1512, 2010.

23. Martinez-Outschoorn UE, Lin Z, Ko YH, Goldberg AF, Flomenberg N, Wang C, Pavlides S, Pestell RG, Howell A, Sotgia F and Lisanti MP: Understanding the metabolic basis of drug resistance: Therapeutic induction of the warburg effect kills cancer cells. Cell Cycle 10: 2521-2528, 2011.

24. Zhao Y, Liu H, Liu Z, Ding Y, Ledoux SP, Wilson GL, Voellmy R, Lin Y, Lin W, Nahta R, et al: Overcoming trastuzumab resistance in breast cancer by targeting dysregulated glucose metabolism. Cancer Res 71: 4585-4597, 2011.

25. Zhong J, Rajaram N, Brizel DM, Frees AE, Ramanujam N, Batinic-Haberle I and Dewhirst MW: Radiation induces aerobic glycolysis through reactive oxygen species. Radiother Oncol 106: 390-396, 2013.

26. Baskar R, Lee KA, Yeo R and Yeoh KW: Cancer and radiation therapy: Current advances and future directions. Int J Med Sci 9: 193-199, 2012.

27. Le A, Cooper CR, Gouw AM, Dinavahi R, Maitra A, Deck LM, Royer RE, Vander Jagt DL, Semenza GL and Dang CV: Inhibition of lactate dehydrogenase A induces oxidative stress and inhibits tumor progression. Proc Natl Acad Sci USA 107: 2037-2042, 2010. 